\title{
Inverse planning in three-dimensional conformal and intensity-modulated radiotherapy of mid-thoracic oesophageal cancer
}

\author{
${ }^{1} \mathrm{~V}$ W C WU, MPhil, HDCR(T), TDCR, ${ }^{2} \mathrm{~J} S \mathrm{~T}$ SHAM, FRCR and ${ }^{2} \mathrm{D}$ L W KWONG, FRCR \\ ${ }^{1}$ Department of Optometry \& Radiography, Hong Kong Polytechnic University, Hung Hom, Kowloon and ${ }^{2}$ Department of \\ Clinical Oncology, University of Hong Kong, Hong Kong
}

\begin{abstract}
The aim of this study is to demonstrate the use of inverse planning in three-dimensional conformal radiation therapy (3DCRT) of oesophageal cancer patients and to evaluate its dosimetric results by comparing them with forward planning of 3DCRT and inverse planning of intensity-modulated radiotherapy (IMRT). For each of the 15 oesophageal cancer patients in this study, the forward 3DCRT, inverse 3DCRT and inverse IMRT plans were produced using the FOCUS treatment planning system. The dosimetric results and the planner's time associated with each of the treatment plans were recorded for comparison. The inverse 3DCRT plans showed similar dosimetric results to the forward plans in the planning target volume (PTV) and organs at risk (OARs). However, they were inferior to that of the IMRT plans in terms of tumour control probability and target dose conformity. Furthermore, the inverse 3DCRT plans were less effective in reducing the percentage lung volume receiving a dose below 25 Gy when compared with the IMRT plans. The inverse 3DCRT plans delivered a similar heart dose as in the forward plans, but higher dose than the IMRT plans. The inverse 3DCRT plans significantly reduced the operator's time by 2.5 fold relative to the forward plans. In conclusion, inverse planning for 3DCRT is a reasonable alternative to the forward planning for oesophageal cancer patients with reduction of the operator's time. However, IMRT has the better potential to allow further dose escalation and improvement of tumour control.
\end{abstract}

Advancement in radiotherapy technologies allows sophisticated radiation dose delivery, including the use of intensity-modulated radiotherapy (IMRT). This is too complicated to be planned by conventional forward planning techniques and inverse planning has emerged as a solution [1-3]. Inverse planning differs from conventional forward planning in that the optimization work is performed by computer algorithms that optimize according to the pre-set dose constraints of the targets and organs at risk (OARs). The potential advantages of inverse planning are less dependence or human planning efforts and reduction of planning time.

With the advancement of computer technology, sophisticated inverse planning algorithms which are able to generate highly complex treatment plans have been developed [4-6]. Currently, inverse planning is mainly employed in IMRT, which is a highly conformal radiotherapy technique achieved by carefully controlling the intensities of individual beamlets within a radiation beam $[7,8]$.

From the perspective of availability of facilities and expertise, three-dimensional conformal radiation therapy (3DCRT) using conventional static beams is more widely available. Compared with IMRT, 3DCRT has the advantages of a more straightforward dose delivery method, which does not require extra equipment and complicated verification procedures. However with highly complex 3DCRT plans forward planning becomes increasingly difficult. It demands considerable experience on the part of the planner and planning time and yet the full potential of 3DCRT may not be realized even with the most experienced

Received 7 March 2003 and in final form 11 December 2003, accepted 3 February 2004. planner. Therefore inverse planning is potentially useful in further improving 3DCRT.

Recent studies on IMRT have reported that it was able to offer better overall dosimetric results compared with traditional radiotherapy techniques $[9,10]$. However tumours which are not in close proximity to critical normal structures do not require a high degree of dose conformity. In these situations, the use of 3DCRT may be already adequate to achieve similar results without the need for additional resources required by IMRT. Inversely planned 3DCRT has the potential to serve as an intermediate option between conventional 2D radiotherapy techniques and IMRT. The aim of this study is to evaluate the relative merits of 3DCRT with inverse planning, 3DCRT with forward planning and IMRT in terms of dosimetric results and planning time. Patients with mid-thoracic oesophageal cancer were chosen to be the subjects of this study, as both 3DCRT and IMRT have been reported to produce promising results for this cancer [11-13].

\section{Methods and materials}

15 patients with stage $\mathrm{II}_{\mathrm{A}-\mathrm{B}}$ tumours in the mid-thoracic oesophagus (AJCC 1997) were randomly recruited. For each patient, the gross tumour volume (GTV), planning target volume (PTV) and the OARs, such as the spinal cord, heart, left and right lungs were delineated in the corresponding CT slices. Each patient was planned using the three planning methods, forward and inverse planning 3DCRT and inverse planning IMRT, using the same treatment planning system (FOCUS Version 3.0 CMS Inc.). To 
facilitate dosimetric comparison, $60 \mathrm{~Gy}$ was prescribed to the centre of the PTV and a common dose endpoint was set as $95 \%$ of the PTV receiving $58 \mathrm{~Gy}$. To eliminate interoperator variations, the same operator computed all the treatment plans.

The computation of the forward 3DCRT plan was based on local planning criteria. It consisted of three beams, one anterior and two posterior-oblique chest beams, with their beam angles, aperture, weight and wedges individually optimized using the manual iterative approach.

The inverse 3DCRT plans were generated by first inputting the dose constraints for the target volumes and OARs (Table 1), which included the dose prescriptions plus their importance factors. The "simplex" autooptimization algorithm was employed to optimize the beam number, orientation, aperture and weight for the 3DCRT plans.

Similarly, the inverse IMRT plans were generated by entering the dose constraints and importance factors for the target volumes and OARs (Table 2). The minor differences in some of the parameters relative to those for 3DCRT were due to the difference in the algorithms between 3DCRT and IMRT inverse planning. In addition, the overlap priority was required for the IMRT plans. This factor governs the priority of consideration during the optimization process when two or more structures overlap. The optimization algorithm used for IMRT was the "Simultaneous interactive inverse treatment planning (SIITP)" [14], which supported the "step and shoot" IMRT dose delivery mode. The optimization was based on the intensity map and levels with the determination of multileaf collimator (MLC) sequence for each beam. Unlike inverse planning in 3DCRT, the number and angles of the beams for the IMRT plans needed to be determined first. In this study, a 5 equi-angled beam arrangement was used with beam angles of $0^{\circ}$ (vertical down beam), $72^{\circ}, 144^{\circ}, 216^{\circ}$ and $288^{\circ}$. An equi-angled beam arrangement was suggested for PTVs that are situated roughly in the central part of the body like the

Table 1. Dose prescription and importance factors of the planning target volume (PTV) and organs at risk in the inverse planning for $3 \mathrm{D}$ conformal radiotherapy

\begin{tabular}{lllll}
\hline Structures & $\begin{array}{l}\text { Min. dose } \\
\text { limit (Gy) }\end{array}$ & $\begin{array}{l}\text { Max. dose } \\
\text { limit (Gy) }\end{array}$ & $\begin{array}{l}\text { Mean dose } \\
\text { limit (Gy) }\end{array}$ & $\begin{array}{l}\text { Importance } \\
\text { factor }\end{array}$ \\
\hline CTV & 58 & 68 & & 80 \\
PTV & 55 & 65 & & 80 \\
Spinal cord & & 40 & 20 & 80 \\
Left lung & 50 & 25 & 50 \\
Right lung & 50 & 2 & 50 \\
Heart & 55 & 30 & 40 \\
\hline
\end{tabular}

oesophagus and prostate. Nutting et al [13], who conducted a dosimetric study on IMRT of oesophageal cancers using 3-9 equi-spaced beams, provided a reference beam arrangement for this study. The beam parameters included in the optimization were the beam size, weight and the configuration of MLC segments.

All three sets of treatment plans were evaluated using the same set of evaluation parameters, which complied with the evaluation criteria recommended in ICRU Report 62 [15]. The evaluation parameters included the conformity index (CI), the homogeneity index (HI) and tumour control probability (TCP), the normal tissue complication probability (NTCP) for the OARs, and the "patient averaged" dose-volume histogram (DVH) for all the structures. The conformity index was the ratio of the $95 \%$ isodose volume to the PTV excluding any factional volume of the PTV receiving a dose below 95\% IL. The HI was the ratio of the maximum dose deviation (maximum minus minimum) to the mean dose within the PTV. For the evaluation of doses to the OARs, the spinal cord, as a serial organ, the maximum dose was used. For the lungs, the V25 was used, which was the percentage volume of the organ receiving $25 \mathrm{~Gy}$ or above as $25 \mathrm{~Gy}$ is the TD50/5 for radiation-induced pneumonitis of lungs.

In addition to the dosimetric parameters, the planning time for the completion of each plan was recorded and compared. The planning time was defined as the time that the operator spent at the planning workstation to complete a treatment plan but excluding the process of structure delineation, which was common to all plans.

\section{Results}

With minor refinements of the optimization criteria, including the maximum dose limits and their relative importance factors for a few patients, the inverse plans for 3DCRT and IMRT were successfully generated. The number of radiation beams employed in the optimized 3DCRT plans ranged from 6 to 16, with a median of 8 beams.

\section{Planning target volume}

All three planning techniques produced acceptable dose distributions to the PTV, with doses within $-5 \%$ and $+7 \%$ of the prescribed dose as recommended by ICRU Report 50 on PTV dose homogeneity [16]. The PTV DVHs for all three sets of plans were similar (Figure 1), however the IMRT plans displayed a higher maximum dose than the 3DCRT plans. Dose conformity was described in terms of the CI. Differences between the forward (For3D) and

Table 2. Dose prescription and importance factors of the planning target volume (PTV) and organs at risk in the inverse planning for intensity-modulated radiotherapy

\begin{tabular}{llllll}
\hline & Minimum (Gy) & Maximum (Gy) & Goal dose (Gy) & Importance factor & Overlapping priority \\
\hline CTV & 58 & 68 & 63 & 100 & 1 \\
PTV & 55 & 64 & 60 & 90 & 2 \\
Spinal cord & & & 20 & 3 & 4 \\
Left lung & 50 & & 20 & 5 & 6 \\
Right lung & 50 & & 30 & \\
Heart & 55 & & & \\
\hline
\end{tabular}


PTV

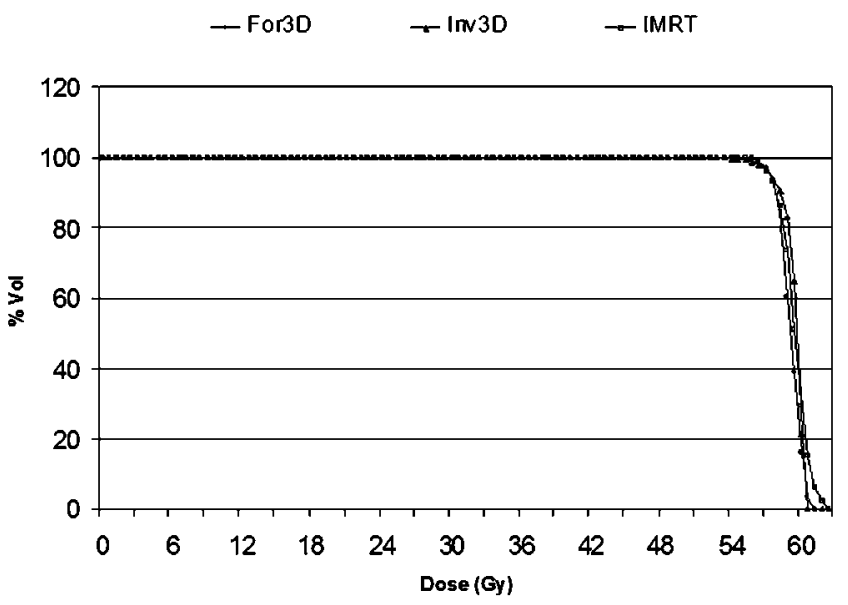

Figure 1. Comparison of dose-volume histograms of planning target volume (PTV). For3D, forward 3DCRT; Inv3D, inverse 3DCRT.

inverse 3DCRT plans (Inv3D) were not statistically significant $(p=0.207)$ but the CI of the inverse 3DCRT plans was significantly lower $(p=0.008)$ than that for IMRT (Table 3 ). There was no statistically significant difference between the three plans in terms of dose homogeneity (HI). The inverse 3DCRT plans presented with higher TCP than the forward plans $(p=0.010)$, but they were lower than the IMRT plans $(p=0.009)$.

\section{Organs at risk}

For the spinal cord, the inverse 3DCRT plans demonstrated comparable maximum dose to that of the forward
Spinal Cord

$\rightarrow$ For3D $\rightarrow \ln 33 \mathrm{D} \rightarrow-$ IMRT



Figure 2. Comparison of dose-volume histograms of the spinal cord. For3D, forward 3DCRT; Inv3D, inverse 3DCRT.

plans $(p=0.480)$ but significantly higher dose than the IMRT plans $(<0.001)$ (Table 4$)$. However with regards to the NTCP, the differences were marginal. A comparison of their mean DVHs is shown in Figure 2.

For the left lung, V25 and NTCP values were statistically the same for For3D and Inv3D $(p=0.478)$, however they were significantly higher than for IMRT $(p=0.001)$. For the right lung, V25 values for Inv3D and For3D $(p=0.058)$ and for Inv3D and IMRT $(p=0.096)$ were statistically the same. NTCP values were significantly lower $(p=0.031)$ and the same $(p=0.539)$ for Inv3D with respect to For3D and IMRT, respectively (Table 4). Comparisons of the mean DVHs of the left and right lungs are shown in Figures 3 and 4, respectively.

The heart received similar doses from both Inv3D and

Table 3. Comparison of the planning target volume dose parameters among the three planning techniques

\begin{tabular}{llllll}
\hline & $\begin{array}{l}\text { Forward 3DCRT } \\
\text { (For3D) }\end{array}$ & $\begin{array}{l}\text { Inverse 3DCRT } \\
\text { (Inv3D) }\end{array}$ & $\begin{array}{l}\text { Inverse IMRT } \\
\text { (IMRT) }\end{array}$ & \multicolumn{2}{l}{ Paired $t$-test, $p$ value } \\
\cline { 5 - 6 } & & $0.62 \pm 0.07$ & $0.68 \pm 0.04$ & 0.207 & Inv3D vs For3D \\
\hline Average CI & $0.63 \pm 0.04$ & $0.17 \pm 0.03$ & $0.19 \pm 0.04$ & 0.184 & 0.008 \\
Average HI & $0.19 \pm 0.03$ & $71.8 \pm 4.4$ & $74.7 \pm 2.4$ & 0.010 & 0.378 \\
Average TCP & $70.1 \pm 4.0$ & & & 0.009 \\
\hline
\end{tabular}

CI, conformity index; HI, homogeneity index; TCP, tumour control probability; IMRT, intensity-modulated radiotherapy; CRT, conformal radiotherapy.

Table 4. Comparison of the dose parameters for the organs at risk among the three planning techniques

\begin{tabular}{|c|c|c|c|c|c|}
\hline & \multirow{2}{*}{$\begin{array}{l}\text { Forward 3DCRT } \\
\text { (For3D) }\end{array}$} & \multirow{2}{*}{$\begin{array}{l}\text { Inverse 3DCRT } \\
\text { (Inv3D) }\end{array}$} & \multirow{2}{*}{$\begin{array}{l}\text { Inverse IMRT } \\
\text { (IMRT) }\end{array}$} & \multicolumn{2}{|c|}{ Paired $t$-test, $p$ value } \\
\hline & & & & Inv3D vs For3D & Inv3D vs IMRT \\
\hline \multicolumn{6}{|l|}{ Spinal cord } \\
\hline Average max. dose (Gy) & $42.9 \pm 5.2$ & $43.6 \pm 6.4$ & $36.1 \pm 2.0$ & 0.480 & $<0.001$ \\
\hline Average NTCP & $0.6 \pm 0.5$ & $0.9 \pm 0.5$ & $0.6 \pm 0.3$ & 0.046 & 0.052 \\
\hline \multicolumn{6}{|l|}{ Left lung } \\
\hline Average V25 (\%) & $40.4 \pm 16.0$ & $45.6 \pm 11.8$ & $21.0 \pm 15.0$ & 0.478 & 0.001 \\
\hline Average NTCP & $5.8 \pm 3.8$ & $5.4 \pm 3.1$ & $3.1 \pm 3.0$ & 0.651 & 0.019 \\
\hline \multicolumn{6}{|l|}{ Right lung } \\
\hline Average V25 (\%) & $45.8 \pm 14.8$ & $38.4 \pm 10.1$ & $30.2 \pm 18.8$ & 0.058 & 0.096 \\
\hline Average NTCP & $7.2 \pm 4.5$ & $4.8 \pm 3.2$ & $4.6 \pm 4.1$ & 0.031 & 0.539 \\
\hline \multicolumn{6}{|l|}{ Heart } \\
\hline Average mean dose (Gy) & $28.5 \pm 13.6$ & $29.0 \pm 14.8$ & $22.2 \pm 13.1$ & 0.720 & 0.058 \\
\hline Average NTCP & $1.5 \pm 1.2$ & $2.2 \pm 1.8$ & $1.4 \pm 1.3$ & 0.294 & 0.022 \\
\hline
\end{tabular}

CRT, conformal radiotherapy; IMRT, intensity-modulated radiotherapy; NTCP, normal tissue complication probability. 
Left Lung

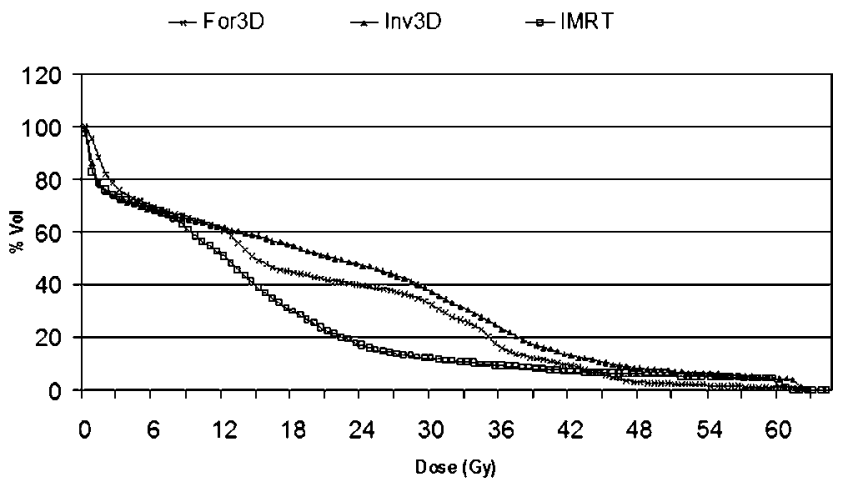

Figure 3. Comparison of dose-volume histograms of the left lung. For3D, forward 3DCRT; Inv3D, inverse 3DCRT.

\section{Right Lung}

$\rightarrow$ For3D $\rightarrow$ Inv3D $\rightarrow$ IMRT

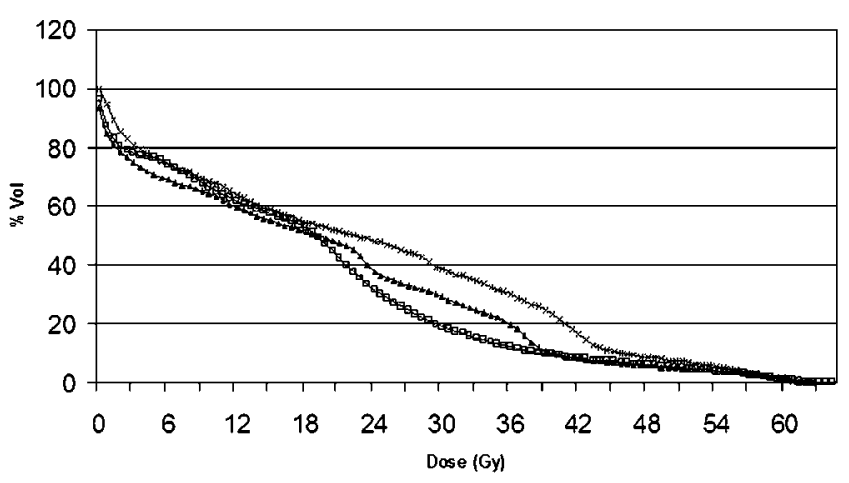

Figure 4. Comparison of dose-volume histograms of the right lung. For3D, forward 3DCRT; Inv3D, inverse 3DCRT.

For3D plans $(p=0.720)$. The corresponding NTCP values were also statistically equivalent $(p=0.294)$. The average mean dose to the heart from IMRT was lower than for Inv3D but the difference was not found to be statistically significant $(p=0.058)$. On the other hand, the NTCP was significantly lower when using the IMRT $(p=0.022)$ (Table 4). Heart DVHs can be seen in Figure 5.

\section{Planning time}

The average planning time was the shortest at $15.1 \mathrm{~min}$ for Inv3D. The time was statistically longer for For3D planning $(p<0.001)$ but not for IMRT planning $(p=0.107)$ (Table 5).

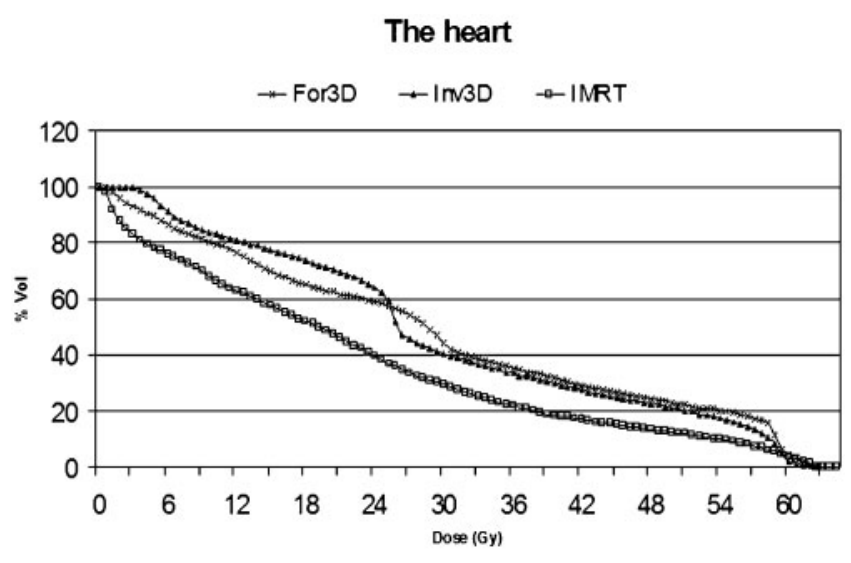

Figure 5. Comparison of dose-volume histograms of the heart. For3D, forward 3DCRT; Inv3D, inverse 3DCRT.

\section{Discussion}

In terms of dosimetric results, the inverse 3DCRT plans demonstrated similar patterns to those of the forward plans for both PTV and OARs. The slightly higher TCP of the inverse 3DCRT plans over the forward plans would not give overall clinical significance between the two techniques because the TCP difference was small $(1.7 \%)$ and the rest of the dose parameters were similar.

All three techniques were able to keep the maximum spinal cord dose below its tolerance of $45 \mathrm{~Gy}$ and able to keep the NTCP of the organ below $1 \%$. Therefore the risk of radiation-induced myelitis by the three techniques was negligible. The reason for the higher spinal cord dose in the inverse 3DCRT plans than the forward plan was that the 3-field forward plans were produced with greater emphasis on sparing the spinal cord, which was only irradiated by the anterior chest field. On the other hand, for the inverse 3DCRT plans, all the structures were considered together during the auto-optimization process. This resulted in a larger proportion of beams hitting the spinal cord.

Compared with the IMRT plans, the inverse 3DCRT plans produced an inferior target dose conformity and TCP. As mentioned before, despite the fact that the 3DCRT plans gave a higher maximum spinal cord dose, the clinical effect would not be significant since both of them were below the organ's tolerance. With regard to the lungs, there was considerable reduction in the lung volume which received a dose below $25 \mathrm{~Gy}$ in the IMRT plans compared with the inverse 3DCRT plans $(24.6 \%$ and $18.2 \%$ for the left and right lungs, respectively). This would make a difference in the volume of lung that might develop radiation complications between the two techniques. As for the heart, the IMRT plans were able to reduce the mean dose and in theory the NTCP relative to the inverse 3DCRT plans. Added to the information

Table 5. Comparison of the planning time among the three planning techniques

\begin{tabular}{lllll}
\hline & $\begin{array}{l}\text { Forward 3DCRT } \\
\text { (For3D) }\end{array}$ & $\begin{array}{l}\text { Inverse 3DCRT } \\
\text { (Inv3D) }\end{array}$ & $\begin{array}{l}\text { Inverse IMRT } \\
\text { (IMRT) }\end{array}$ & $\begin{array}{l}\text { Paired } t \text {-test, } p \text { value } \\
\text { Inv3D vs For3D }\end{array}$ \\
\hline Average planning time (min) & $36.5 \pm 10.4$ & $15.1 \pm 7.5$ & $19.2 \pm 5.9$ & $<0.001$ \\
\hline
\end{tabular}

CRT, conformal radiotherapy; IMRT, intensity-modulated radiotherapy. 
observed in the DVHs (Figure 5), difference in the risk of percarditis could be significant. Based on the dose delivered to the OARs for the IMRT plans, it would be possible to escalate the dose with the IMRT plans to the PTV to a degree that would produce the same complication risks of the OARs as for the 3DCRT plans.

The main reason for the better dosimetric results in the IMRT plans was that the optimization algorithm employed worked on intensity levels of individual beamlets within a radiation beam. The SIITP algorithm used in the IMRT plans, which belongs to one version of the stochastic algorithm, works with a "score function" using the "sum of squares dose deviation". A stochastic algorithm in inverse planning is referred to as an "iterative optimization algorithm" that, basically, randomly finds new positions in the search space at each iteration step. The score of each iteration, which was calculated according to the prescribed dose constraints and importance factors, was compared with that for the next iteration. During the optimization, the system looks for the lowest score and stops when the convergence criterion is met. This was more sophisticated than the "simplex" algorithm used in the inverse 3DCRT plans and is more effective in producing tightly conformal dose distributions to the target volumes. The "simplex" inverse planning algorithm used for the 3DCRT plan optimization belongs to one model of the linear programming algorithms, which were believed to be a robust model for linear problems [17]. The solution to the inverse problem was to maximize the PTV dose and minimize the OAR doses under the set of planning criteria prescribed prior to the optimization process. The optimal plan was achieved by solving equations involving all the treatment parameters.

In terms of planning time, inverse planning demonstrated significant advantage in reducing the planner's time, when compared with conventional forward planning for 3DCRT. On average, the inverse 3DCRT plans shortened the planner's time by 2.5 fold compared with the forward plans, and the planning time for inverse 3DCRT planning was comparable with that of the IMRT plans. In addition, there are two other practical issues that need to be considered. First, more time is required in the verification and dose delivery processes of IMRT but this was not assessed in this study. Second, the number of beams used for the inverse 3DCRT plans is larger than for the IMRT and forward plans. However, this will not necessarily increase the overall treatment time compared with IMRT because the delivery of 3DCRT beams does not need to wait for the sequential movement of the MLC segments as in the case of IMRT, and therefore the time spent on each beam is much shorter. Furthermore, with the availability of computerized linear accelerators, and by restricting the inverse 3DCRT plans to co-planar beams that share a common set up point, the increase in treatment time due to increase in beam number can be minimized.

\section{Conclusion}

Owing to the complexity of treatment planning for sophisticated conformal radiotherapy, inverse planning is becoming increasingly important. Forward planning for 3DCRT, which requires the consideration of a large quantity of inter-related treatment parameters, is time and labour consuming. Any alternatives that can improve this situation and at the same time produce better dosimetric outcomes would be welcome.

In conclusion, with regards to the radical radiotherapy of mid-thoracic oesophageal cancer patients, inverse planning for $3 \mathrm{DCRT}$ is able to produce reasonable alternative plans to the forward planning approach with reduction of planner's time. However, further dose escalation and an improvement of tumour control may need to rely on IMRT treatment plans.

\section{References}

1. Mackie TR, Holmes TW, Deasy JO, Reckwert PJ. New trends in treatment planning. Phys Med Biol 1994;39A(1):480.

2. Barth NH. An inverse problem in radiation therapy. Int $\mathbf{J}$ Radiat Oncol Biol Phys 1990;18:425-31.

3. Oelfke $U$, Bortfield $T$. Inverse planning for $\mathrm{x}$-ray rotation therapy: a general solution of the inverse problem. Phys Med Biol 1999;44:1089-104.

4. Li RP, Yin FF. Optimisation of inverse treatment planning using a fuzzy weight function. Med Phys 2000;27:691-700.

5 . Webb S. Optimisation of conformal radiotherapy dose distributions by simulated annealing. Phys Med Biol 1989; 34:1349-69.

6. Lind BK. Properties of an algorithm for solving the inverse problem. Inverse problem 1990;6:415-26.

7. Teh BS, Woo SY, Butler EB. Intensity modulated radiation therapy (IMRT): a new promising technology in radiation oncology. The Oncologist 1999;4:433-42.

8. Webb S, Convery DJ, Evans PM. Inverse planning with constraints to generate smoothed intensity modulated beams. Phys Med Biol 1998;43:2785-94.

9. Verhey LJ. Comparison of three-dimensional conformal radiation therapy and intensity modulated radiation therapy systems. Semin Radiat Oncol 1999;19:78-98.

10. Purdy JA. 3D treatment planning and intensity-modulated radiation therapy. Oncology 1999;13:155-68.

11. Guzel Z, Bedford JL, Childs PJ, Nahum AE, Webb S, et al. A comparison of conventional and conformal radiotherapy of the oesophagus: work in progress. Br J Radiol 1998;71: 1076-82.

12. Bedford JL, Viviers L, Guzel Z, Child PJ, Webb S, et al. A quantitative treatment planning study evaluating the potential of dose escalation in conformal radiotherapy of the oesophagus. Radiother Oncol 2000;57:183-93.

13. Nutting CM, Bedford JL, Cosgrove VP, Tait DM, et al. A comparison of conformal and intensity-modulated techniques for oesophageal radiotherapy. Radiother Oncol 2001;61: $157-63$.

14. Xing L, Hamilton RJ, Spelbring D, Pelizzari CA, et al. Fast iterative algorithms for three-dimensional inverse treatment planning. Med Phys 1998;25:1845-9.

15. Wambersie A, Landberg T. ICRU Report 62: Prescribing, recording and reporting photon beam therapy (Supplement to ICRU Report 50) 1999

16. International Commission on Radiation Units and Measurements. ICRU Report 50. Prescribing, recording, and reporting photon beam therapy. Bethesda. 1993.

17. Newman F. Conventional inverse planning. Sphere of Influence 2000;4:8-11. 